\title{
$\widehat{A}$ Madridge \\ madridge Journal of Dermatology \& Research \\ Interconnecting Scientific World
}

Research Article

Open Access

\section{A Methodological Approach for Analysis of Melanoma Images}

\author{
Sudhakar Singh ${ }^{1 \star}$ and Shabana Urooj ${ }^{2}$ \\ Department of Electrical Engineering, Gautam Buddha University, Greater Noida, India
}

Article Info

\author{
*Corresponding author: \\ Sudhakar Singh \\ Department of Electrical Engineering \\ Gautam Buddha University \\ Greater Noida \\ India \\ E-mail: sudhakarsingh86@gmail.com
}

Received: October 25, 2018

Accepted: November 23, 2018

Published: November 29, 2018

Citation: Singh S, Urooj S. A Methodological Approach for Analysis of Melanoma Images. Madridge J Dermatol Res. 2018; 3(2): 83-87. doi: $10.18689 / \mathrm{mjdr}-1000121$

Copyright: @ 2018 The Author(s). This work is licensed under a Creative Commons Attribution 4.0 International License, which permits unrestricted use, distribution, and reproduction in any medium, provided the original work is properly cited.

Published by Madridge Publishers

\begin{abstract}
In the proposed work complete task is divided in three parts. First is preprocess and segment the image. After segmentation different features (texture, color, higher order spectra (HOS)) have been extracted. These extracted features are applied for for the classification. Back-propagation-Artificial Neural Network (ANN) has been used for melanoma image classification. Performances of five different algorithms are compared in terms of accuracy, meantime, min-time and max-time. The accuracy of the proposed technique is $84.42 \%$ which is beter than other exhisting techniques. In the present work a dataset of 950 images of melanoma is used for the classification in which 500 for training and 250 for testing.
\end{abstract}

Keywords: Feature Extraction; Back-Propagation-ANN; Melanoma; Skin Cancer.

\section{Introduction}

Skin cancer is one of the disease in the modern world which causes death to quite a few number of patients. Medical professionals and researchers are trying to find the root cause of the disease and produce effective medicines for the same. Most of the patients don't know about their skin cancer till it is in final stages. The basic diagnosis of a skin cancer is done using visual inspection by a general physician and if any further investigation needed the case is referred to a dermatologist. A biopsy and pathological analysis is carried out to know the type of cancer (Basal Cell Skin Cancer (BCC), Squamous-Cell Skin Carcinoma and Malignat Melanoma). The deadliest among these three are Malignat Melanoma. It has an assymetrical area, color variation, irregular border and diameter is often greater than $6 \mathrm{~mm}$. Maligant Melanoma can cause death if it goes undetected or it spreads in the human skin.

Once the CAD analysis has been done, the skin expert can take the decision based upon the classification results [1]. Dermoscopy is a first and foremost extremely helpful standard technique for diagnosing the spiteful cells of skin disease [2,3]. Skin infection is a large amount added unsafe if it is not found in the premature stages [4].

\section{Related Work}

A number of the fuzzy logic techniques examined in preceding research used for color and furthermore skin assessment, including color histogram examination for color marking and skin laceration intolerance [5]. Unfairness, constraint recognition is also defined in skin lesion using fuzzy clustering and texture analysis [5]. The fuzzy clustering segmentation technique is used for the adaptive removing background of skin color for part segmentation [5] and fuzzy c-means clustering considered for pelt scratch analysis [6]. Adaptive fuzzy, c-means improved technique uses local spatial continuity for cluster pattern evaluation $[7,8]$, here skin area segmented by fuzzy estimation grade modeling [8]. Scheduled starting the foundation of artificial intelligence, soft computing, and image 
processing, used for design as of a number of authors have furthermore, the addition of area understanding concerning the individuality of objects increases the segmentation precision $[9,10]$. The fuzzy rules are functional among every single point of thoughtfulness (applicant points used for shape detection) single individuals substantial the rule sets are reserved for investigation, by applying a number of criteria of decimation. Based deceitful on the segmented regions of interest, the morphological features and spatial distribution are constructed. These features are classified by a multi-class support vector machine classifier [11,12]. Melanoma parameters including Asymmetry, Border, color, diam, (ABCD) it checked by the lesion Image analysis tools.

\section{Methodology}

In the proposed work a set of 950 melanoma images is used. All Images having the same size (1022x762), taken from International Skin Imaging Collaboration [13]. Color features, texture features, HOS features [14] are examined. Back propagation-ANN is used, for performanceanalysis of different algorithms. Block feature, magnitude of patches, angle of patches and normalized feature of cancerous part is calculated. MSE and Accuracy are measured and compared with other existing techniques.

\section{Feature Extraction}

Feature selection eliminates the dimensionality of data by selecting only a subset of deliberate features to generate a model. Feature choice is preferable to feature adaptation when the exceptional units and meaning of features are important and the modeling goal is to identify a significant subset.

\section{Color Features}

A digit of major color, features has been projected in the literatures, including color histogram $[15,16]$, color moments (CM) [17], color coherence vector (CCV) and color correlogram [18], etc. Among them, CM is one of the simplest yet very effective features. The general moments are mean, standard deviation and skewness; the corresponding calculation can be defined as follows:

$\mu_{i}=\frac{1}{n} \sum_{j=1}^{n} X_{i j}$

$\sigma_{i}=\sqrt{\frac{1}{n} \sum_{i=1}^{n}\left(X_{i j}-\mu_{i}\right)^{2}}$

$\gamma_{i}=\sqrt[3]{\frac{1}{n} \sum_{i=1}^{n}\left(X_{i j}-\mu_{i}\right)^{2}}$

Where $X_{i j}$ is the color value of the $i^{\text {th }}$ color component of the $\mathrm{j}$-th image pixel and $\mathrm{n}$ is the total number of pixels in the image. $\mu_{i}, \sigma_{i}, \gamma_{i}(\mathrm{i}=1,2,3)$ denote the mean, standard deviation and skewness of each channel of an image respectively [20].

\section{Texture Features}

Texture is a quite helpful classification for an extensive series of image. A number of techniques have been projected to remove texture features. Texture features are extracted through computing the pixel statistics or finding the local pixel structures in the original image domain, while the latter transforms an image into the frequency domain and then calculates feature from the transformed image. Gabor filter [19] has been widely used in image texture feature extraction. Thus, texture features can be extracted from this group of energy distributions [20]. Given an input image I $(x, y)$, Gabor wavelet transform convolves I $(x, y)$ with a set of Gabor filters of unlike spatial frequencies as well as orientations. A twodimensional Gabor function $\mathrm{g}(\mathrm{x}, \mathrm{y})$ can be defined as follows.

$$
g(x, y)=\frac{1}{2 \pi \sigma_{X} \sigma_{y}} \exp \left[-\frac{1}{2}\left(\frac{x^{2}}{\sigma_{X}^{2}}+\frac{y^{2}}{\sigma_{y}^{2}}\right)+2 \pi j W_{X}\right]
$$

Where $\sigma_{X}$ and $\sigma_{y}$ are the scaling parameters of the filter, $\mathrm{W}_{\mathrm{x}}$ is the center frequency, and $\theta$ determines the orientation of the filter. Figure 1 shows the Gabor function in the spatial domain.

\section{HOS Features}

Higher order spectra features are used to investigate the nonlinear and dynamic nature of the given signal [21]. The random distribution of pixels of lesions and nonlinear behavior among the frequency components supports us to use HOS features. Here extracted the HOS features for every $1^{\circ}$ of the Radon transform between $0^{\circ}$ and $180^{\circ}$. Eq. (5) represents the bispectrum of signal.

$x(k)$ for $|\omega 1| \leq,|\omega 2| \leq$ and $|\omega 1+\omega 2| \leq$.

$\mathrm{B}(\omega 1, \omega 2)=\mathrm{X}(\omega 1) \mathrm{X}(\omega 2) \mathrm{X} *(\omega 1+\omega 2)$

Mean of spectral magnitude of power spectrum:

$M_{\text {avg }}=\frac{1}{n} \sum_{i=1}^{n=1} X(i)$

Here X(i) is the Fourier Transform of input signal. Mean of spectral magnitude for HOS.

\section{Contrast}

$C o=\sum_{m} \sum_{n}(m-n)^{2} c(m-n)$

Homogeneity

$H o=\sum_{m} \sum_{n} c \frac{(m, n)}{1+(m-n)^{2}}$

\section{Back Propagation Neural Network}

Back propagation is the extended form of the WidrowHoff learning rule in the direction of multiple-layer networks and nonlinear differentiable transfer functions. Input vectors and the analogous object vectors are used to train a network awaiting it can estimate a function, link input vectors by means of specific output vectors. The BPN explained here contains three layers. These layers are input, 
hidden, and output layers. For the duration of the training phase, the training data are given to the input layer. The data is passed through the hidden layer and then to the output layer. This is called the forward pass of the back propagation algorithm [1].

\section{Algorithm}

Back propagation Neural Network algorithm is important for the analysis of image processing. The algorithm is taken from Simon Hykin [22].

\section{Preliminaries}

- Training set: $\left(A_{p^{\prime}} B_{p}\right), 1,1 \leq p \leq P$

- $\mathrm{P}=$ input number

- $x_{p}=\left(x_{p}, 0, \ldots . . . x_{p}, N\right) / /$ Input

- $A_{p}=\left(A_{p^{\prime}}, \ldots, \ldots . . A_{p^{\prime}} K\right) / /$ output for $d_{p}$

- $\mathrm{N}=$ space input dimension

- $\mathrm{K}$ = output of neurons number

- $B_{p}=\left(B_{p^{\prime}}, \ldots \ldots . . B_{p^{\prime}} K\right) / /$ Actual Output

- $e_{p^{\prime} j}=\left|A_{p, j}-B_{p^{\prime} j}\right| / /$ error

- $x_{p^{\prime} i}:$ value in $i^{\text {th }}$ input node

- $\operatorname{Net}{ }_{\mathrm{j}}^{(1)}=\sum_{i=0}^{n} w_{j, i}^{(1,0)} x_{p, i} \mathrm{j}$ is hidden layer, net input of $\mathrm{j}^{\text {th }}$ node in hidden layer.

- $w^{(i+1, i)}{ }_{k, j}$ : weight from $j^{\text {th }}$ node in $i^{\text {th }}$ layer to $k^{\text {th }}$ node of $(\mathrm{i}+1)^{\text {th }}$ layer

- $B_{p^{\prime} k}=\sigma\left(\sum j \cdot w_{k, j}^{(2,1)} \cdot x_{p, j}^{(1)}\right)$ : Output of $k^{\text {th }}$ node in output layer.

$-x_{p, j}^{(i)}$ : Output of $j^{\text {th }}$ of $i^{\text {th }}$ layer for patterns $x_{p .}$

- net $^{(2)}=\sum_{i=0}^{n} w_{k, i}^{(1,0)} \cdot x_{p, i} \quad k$ is output layer, net output of $\mathrm{k}^{\text {th }}$ node in output layer.

- $\mathrm{x}_{\mathrm{p}, \mathrm{j}}^{(1)}=\sigma\left(\sum_{i=0}^{n} w_{j, i}^{(1,0)} x_{p, i}\right)$ :Output of $\mathrm{j}^{\text {th }}$ node in the hidden layer

- Activation function, logistic sigmoid $\sigma($ net $)=1 /\left(1+e^{\alpha \text { net }}\right)$

- $\delta$ proportional to actual error $\mid$ deck $-o_{k} \mid$ multiplied by the derivative of output node i.e. $\delta_{k}=\left(\right.$ deck $-o_{k} \cdot O k \cdot\left(i-o_{k}\right)$

- $\mu_{\mathrm{j}}$ proportional to "weighted sum of errors" coming to the hidden node from a node in upper layer i.e. $\mu_{j}=\Sigma_{k}$ $\cdot \delta_{k} \mathrm{w}^{(2,1)}{ }_{k, j} \cdot x_{j}^{(1)} \cdot\left(1-x^{(1)}\right)$ Objective: Cost function: Minimize Cumulative Error defined in Equation 10.

$\sum_{p=1}^{p} \operatorname{Err}\left(A_{p}, B_{p}\right)$

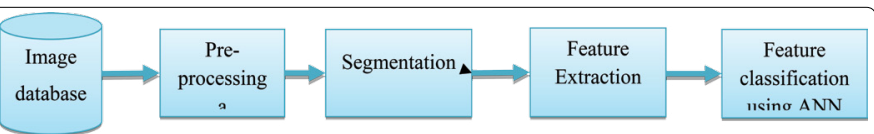

Figure 1. Proposed technique for melanoma classification (a)

(b)

(c)

(d)

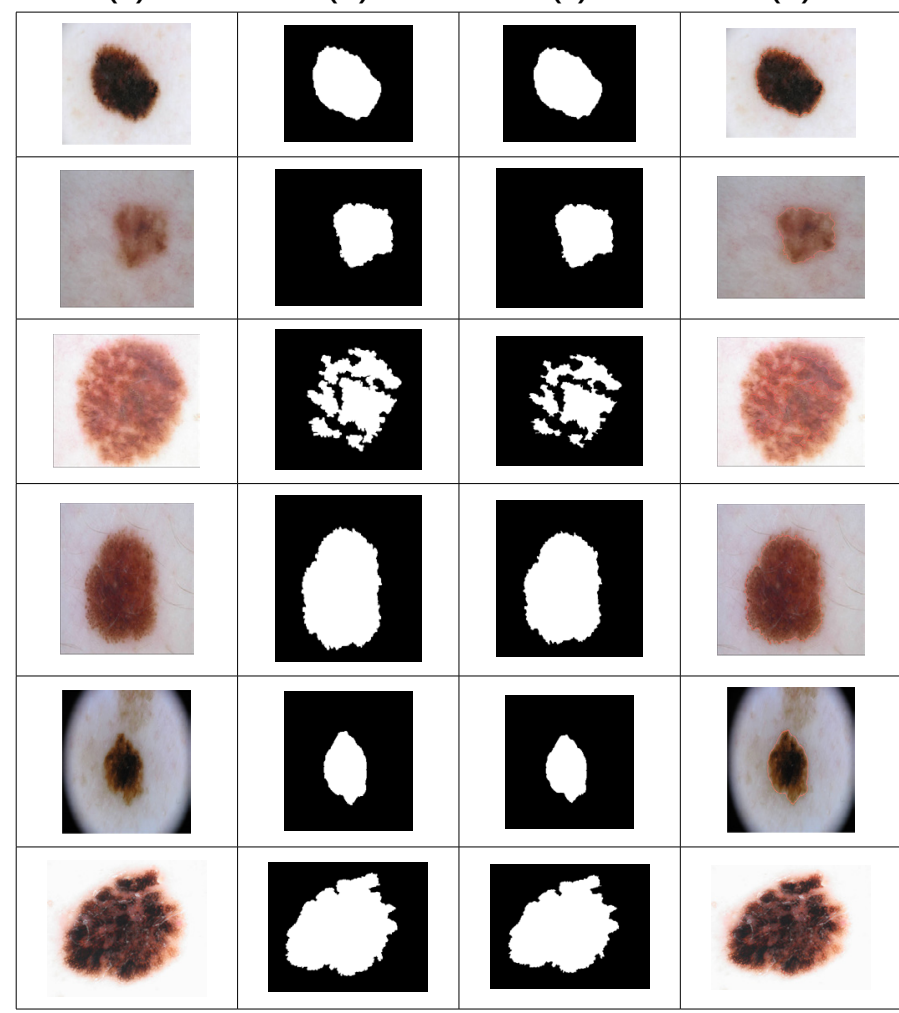

Figure 2. (a) Test Image, (b) Binary Mask, (c) Border Removed, (d) Segmented Image

Table.1 Features of the skin diseases images

\begin{tabular}{|c|l|l|l|l|l|l|l|l|}
\hline $\begin{array}{c}\text { Images } \\
\text { No. }\end{array}$ & Mean & Skewness & $\begin{array}{l}\text { Standard } \\
\text { Deviation }\end{array}$ & Kurtosis & Variance & $\begin{array}{l}\text { Central } \\
\text { Moment }\end{array}$ & Entropy & Threshold \\
\hline 1 & 0.3463 & 3.0962 & 0.5051 & 9.4128 & 0.0864 & 0.4128 & 0.8731 & 0.4823 \\
\hline 2 & 0.3423 & 5.8333 & 0.4766 & 35.0277 & 0.2251 & 0.0961 & 0.3963 & 0.4823 \\
\hline 3 & 0.6348 & 5.1673 & 0.50137 & 27.70155 & 0.2499 & 0.0961 & 0.9111 & 0.4823 \\
\hline 4 & 0.7400 & 4.6948 & 0.5050 & 23.0416 & 0.2500 & 0.0960 & 0.9756 & 0.4823 \\
\hline 5 & 0.4344 & 2.8618 & 0.4977 & 9.1900 & 0.2457 & 0.0956 & 0.5053 & 0.4823 \\
\hline 6 & 0.5436 & 3.3421 & 0.5340 & 22.5462 & 0.2453 & 0.0960 & 0.5467 & 0.0 .4803 \\
\hline
\end{tabular}

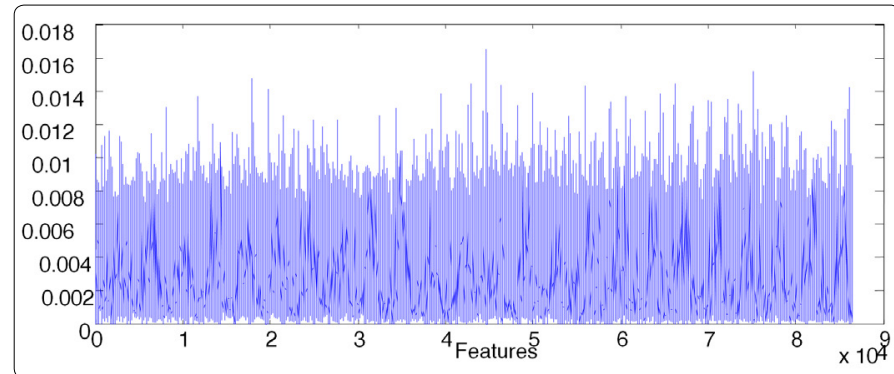

Figure 3. Features of the Melanoma Image

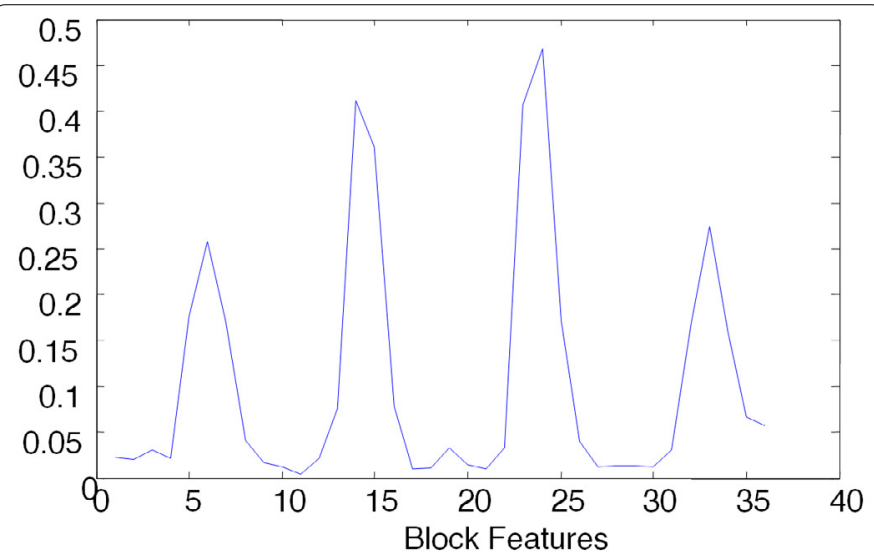

Figure 4. Block Features of cancerous part 


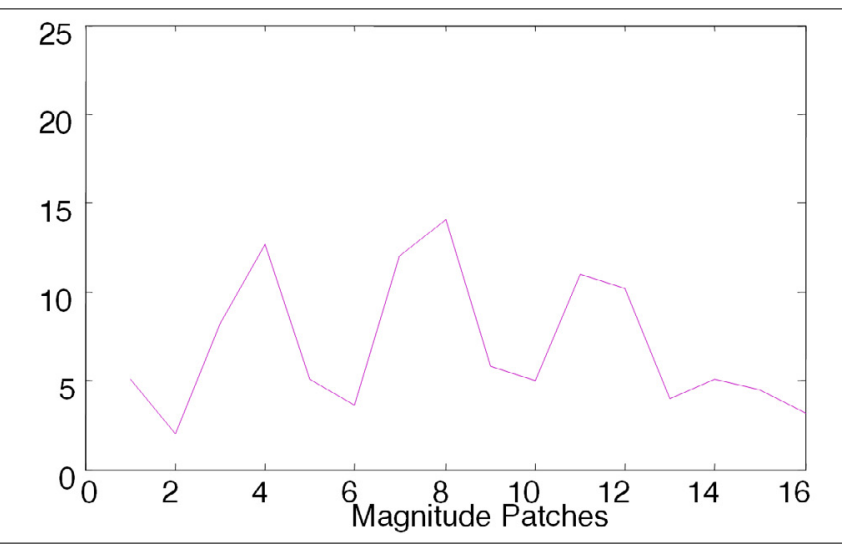

Figure 5. Magnitude Patches of cancerouspart

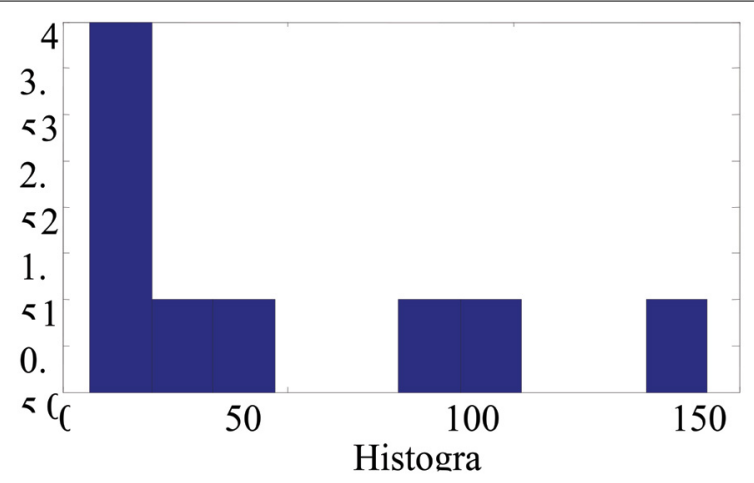

Figure 6. Normalized block features

$$
40
$$

30

20

10

0

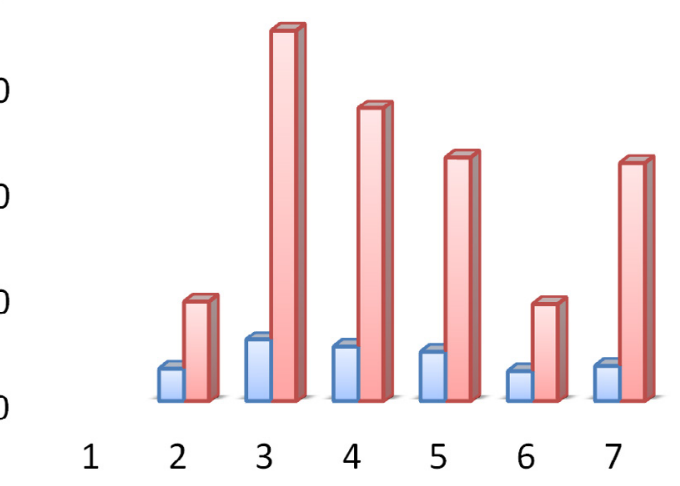

$\square$ Skewness $\square$ Kurtosis

Figure 7. Features of melanoma

$\square$ Mean $\square$ Standard Deviation $\square$ Variance $\backsim$ Central Moment $\square$ Entropy $\square$ Threshold

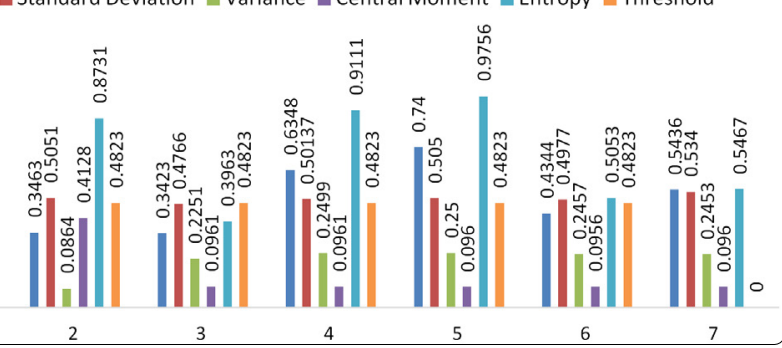

Figure 8. Features of melanoma.

Table 2. Performance of different algorithm

\begin{tabular}{|l|l|l|l|}
\hline Algorithm & Mean Time (sec.) & Min. Time (sec.) & Max. Time (sec.) \\
\hline CGP & 5.00 & 3.10 & 8.03 \\
\hline CGB & 6.30 & 4.03 & 12.59 \\
\hline CGF & 7.62 & 4.46 & 26.05 \\
\hline LM & 14.07 & 7.48 & 26.78 \\
\hline GDX & 28.07 & 26.21 & 27.52 \\
\hline
\end{tabular}

The proposed approach involved in preprocessing, segmentation, feature extraction, feature selection and ANN based classification of the image. The system was tested on a large set of images 950. Primarily, the images used in this study came from different sources. For this reason, care was taken to extract features that are invariant to changes in the imaging conditions. Table 1 shows the statistical features of the different skin diseases. There are six different skin diseases as shown in figure 2 and seven different statistical features are calculated for the classification of skin diseases images. In this paper different feature (color, texture, HOS) for the melanoma image, is discussed. Figure3 depicts texture feature calculated for melanoma image. Figure 4 reprents block features for the melanoma images, these block features are features of cancerous part of the melanoma image. figure 5 represents magnitude Patches of cancerouspart. Figure 6 shows the normalized features of the cancerous part of melanoma images. figure 7,8 brief about statistical features ofmelanoma images. From figure 7 and figure 2 it is observed that the skewness and kurtosis is highest for the second image of column (a), and lowest for first image of column (a). From figure 2 and figure 8, highest value of entropy for image 3 and lowest for the image 2 of column (a). Threshold value of all the images are nearly same. Central Moment of the first image of column is highest. Variance of the third image is highest. Mean valvue of the third image of column is highest.

Table 3. Performance of proposed technique with other existing techniques.

\begin{tabular}{|c|c|c|c|c|c|c|}
\hline Authors (year) & Dataset & $\begin{array}{l}\text { Data } \\
\text { size }\end{array}$ & Features & \begin{tabular}{|l|} 
Feature \\
selection \\
technique
\end{tabular} & Classifier & $\begin{array}{l}\text { Performance } \\
\text { measure }\end{array}$ \\
\hline $\begin{array}{l}\text { Messadi et al. (2009) } \\
{[30]}\end{array}$ & Melanoma & 180 & $A B C D$ rule & NA & ANN & $\begin{array}{l}\text { SE: } 67.50 \% ; \\
\text { SP: } 80.50 \% \\
\text { ACC: } 74.50 \%\end{array}$ \\
\hline $\begin{array}{l}\text { Rahman et al. (2010) } \\
{[31]}\end{array}$ & Melanoma 8 & 35 & Texture & Color & $\begin{array}{l}\text { KNN, } \\
\text { NN, } \\
\text { SVM, ML } \\
\text { Gaussian }\end{array}$ & ACC: $75.69 \%$ \\
\hline Faal et al. (2013) [32] & Melanoma & 436 & $\begin{array}{l}\text { Shape, Texture, } \\
\text { Color }\end{array}$ & PCA KNN & $\begin{array}{l}\text { SVM, } \\
\text { LDA }\end{array}$ & $\begin{array}{l}\text { SE: } 83.53 \% \\
\text { ACC: } 80.46 \%\end{array}$ \\
\hline $\begin{array}{l}\text { Pereira et al. (2013) } \\
\text { [33] }\end{array}$ & $\begin{array}{l}\text { Ulcer } \\
\text { Images }\end{array}$ & 172 & Texture, Color & $\begin{array}{l}\text { Wrapper } \\
\text { Algorithm }\end{array}$ & ANN & ACC: 73.80 \\
\hline Proposed Technique & Melanoma & 350 & $\begin{array}{l}\text { Texture, } \\
\text { Color, HOS }\end{array}$ & Color & BPNN & ACC:84.42 \\
\hline
\end{tabular}

The subsequent table summarizes the results of training this network with the five different algorithms. In all cases, the network is trained up to the error is less than 0.001. The fastest algorithm for this problem is the Polak-Ribiére Conjugate Gradient back propagation algorithm. table 2 shows the performance of the different algorithm used in back propagation ANN. Minimum time taken by the CGP algorithm in terms of meantime, min-time, and max-time. CGP algorithm is better than all four algorithm used. Table 3 shows the performance of the proposed technique with other existing technique. The technique has been used for the 950 melanoma images and accuracy of proposed algorithm is $84.42 \%$, which is better than as compared to the other technique.

\section{Conclusion}

In this paper image segmentation, different features (statistical feature, block feature, magnitude features) are extracted and then classification is performed. Performance 
of different training algorithms are observed in which CGP algorithm was better in terms of time consumption. The accuracy of the thechnique is $84.42 \%$ which is better than other three techniques as shown in table 3. In future we plan to incorporate more advanced features related to diagnostic relevance into our system and experiment with other classification techniques.

\section{References}

1. Gutiérrez-Arriola JM, Gómez-Álvarez M, Osma-Ruiz V, Sáenz-Lechón N, Fraile R. Skin lesion segmentation based on preprocessing, thresholding and neural networks. Expert Syst Appl. 2017.

2. Sheha M, Mabrouk M, Sharawy A. Automatic detection of melanoma skin cancer using texture analysis. International Journal of Computer Applications. 2012; 42(20): 22-26. doi: 10.5120/5817-8129

3. Sudhakar S, Urooj S, Singh SP. Analysis of Leukoderma Images Using Neuro-Fuzzy Hybrid Technique. Sensors and Image Processing. 2017; 93101.

4. Zortea M, Flores E, Scharcanski J. A simple weighted thresholding method for the segmentation of pigmented skin lesions in macroscopic images. Pattern Recognit. 2017; 64: 92-104. doi: 10.1016/j. patcog.2016.10.031

5. Ng VTY, Fung BYM, Lee TK. Determining the asymmetry of skin lesion with fuzzy borders. Comput Biol Med. 2005; 35(2): 103-120. doi: 10.1016/j.compbiomed.2003.11.004

6. Rastgoo M, Garcia R, Morel O, Marzani F. Automatic differentiation of melanoma from dysplastic nevi. Comput Med Imaging Graph. 2015; 43: 44-52. doi: 10.1016/j.compmedimag.2015.02.011

7. Celebi ME, Kingravi HA, Uddin B, et al. A methodological approach to the classification of dermoscopy images. Comput Med Imaging Graph. 2007; 31(6): 362-373. doi: 10.1016/j.compmedimag.2007.01.003

8. Chakravorty R, Liang S, Abedini M, Garnavi R. Dermatologist-like feature extraction from skin lesion for improved asymmetry classification in $\mathrm{PH} 2$ database. In Proceedings of the Annual International Conference of the IEEE Engineering in Medicine and Biology Society. EMBS. 2016; doi: 10.1109/EMBC.2016.7591569

9. Barata C, Celebi ME, Marques JS, Rozeira J. Clinically inspired analysis of dermoscopy images using a generative model. Comput Vis Image Underst. 2016; 151: 124-137. doi: 10.1016/j.cviu.2015.09.011
10. Pomponiu V, Nejati H, Cheung NM. Deepmole: Deep neural networks for skin mole lesion classification. In Proceedings - International Conference on Image Processing. ICIP. 2016. doi: 10.1109/ICIP.2016.7532834

11. Kasmi R, Mokrani K, Rader RK, Cole JG, Stoecker WV. Biologically inspired skin lesion segmentation using a geodesic active contour technique. Ski Res Technol. 2016; 22(2): 208-222. doi:10.1111/srt.12252

12. Sudhakar S, Urooj S. Mathematical Modeling of Sensitivity and Specificity for Basal Cell Carcinoma (BCC) Images. Information and Decision Sciences. 2018; 473-480.

13. Matsunaga K, Hamada A, Minagawa A, Koga H. Image Classification of Melanoma, Nevus and Seborrheic Keratosis by Deep Neural Network Ensemble. 1 Isic. 2017: 2-5.

14. Ballerini $L$, Fisher $R$, Aldridge $B$, Rees J. A color and texture based hierarchical K-NN approach to the classification of non-melanoma skin lesions. Color Med Image Anal. 2013; 63-86.

15. Yu L, Chen H, Dou Q, Qin J, Heng PA. Automated Melanoma Recognition in Dermoscopy Images via Very Deep Residual Networks. IEEE Trans Med Imaging. 2017; 36(4): 994-1004. doi: 10.1109/TMI.2016.2642839

16. Faizal Khan Z, Ganapathi N. Texture based automated segmentation of skin lesions using Echo State Neural networks. J Electr Eng Technol. 2017; 12(1): 436-442. doi: 10.5370/JEET.2017.12.1.436

17. Mennesson J, Saint-Jean C, Mascarilla L. Color Fourier-Mellin descriptors for image recognition. Pattern Recognit Lett. 2014; 40(1): 27-35. doi:10.1016/j.patrec.2013.12.014

18. Chen WT, Liu WC, Chen MS. Adaptive color feature extraction based on image color distributions. IEEE Trans Image Process. 2010; 19(8): doi: 10.1109/TIP.2010.2051753

19. Zhan Y, Shen D. Deformable segmentation of 3-D ultrasound prostate images using statistical texture matching method. IEEE Trans Med Imaging. 2006; 25(3): 256-272. doi: 10.1109/TMI.2005.862744

20. Li W, Mao KZ, Zhang H, Chai T. Selection of gabor filters for improved texture feature extraction. In Proceedings - International Conference on Image Processing. ICIP. 2010. doi: 10.1109/ICIP.2010.5653278

21. Urooj S, Singh S. A novel computer assisted approach for diagnosis of skin disease. Computing for Sustainable Global Development (INDIACom). 2015; 1585-1590.

22. Brito R, Fong $S$, Cho K, et al. GPU-enabled back-propagation artificial neural network for digit recognition in parallel. J Supercomput. 2016; 72(10): 3868-3886. doi: 10.1007/s11227-016-1633-y 